\title{
Key activities of competitive intelligence (4)
}

\author{
Marié-Luce Muller \\ mlmuller@ibis.co.za
}

\section{Introduction}

This column examines the basic operational areas or constructs of competitive intelligence (CCI). In the previous columns the planning and focus, collection and analysis constructs of CI were scrutinized and communication or dissemination is now examined as an activity area. It is an activity that closely relates to the structure that exists for CI to work effectively in a company.

CI has earned its rightful place as a strategic management tool in companies. Successful CI capabilities provide more than just knowledge about developments in the competitive environment - it should provide forewarning of opportunities and threats. According to McGonagle and Vella (1990) CI can assist business leaders to make better decisions than their competitors with regard to:

- Competitive activities, e.g. mergers and acquisitions

- Marketing planning, e.g. new product development

- Regulatory issues, e.g. impact of new legislation on the business

- Customer activities, e.g. changing needs and preferences.

CI starts with identifying the company's key intelligence needs or requirements (those issues that keep decision makers awake at night) and then collecting and analysing information that would provide an answer to the intelligence need and finally, disseminating the intelligence to the decision maker for action taking. The operational areas are all important and not one can stand alone - there is no short cut. Omitting any part of the CI process may have dire consequences. For example, leaving out the analysis task will lead to the delivery of un-integrated data summaries to management, while a lack or absence of communication means that intelligence generated becomes worthless.

\section{Recapping the key operational areas and their role and function}

- Planning and focus. CI should only focus on those business issues that are of critical importance for a company to know. These issues are known as key intelligence needs or requirements.

- Collection. It is during this phase that information is collected from a variety of sources for examination and verification during the CI process. Collection comes from a variety of different sources and gathering techniques.

- Analysis. During this phase, information is turned into intelligence through a process of interpretation and the results should be usable in strategic decision making.

- Dissemination. The results of the CI process are disseminated in an appropriate format and at the right time to those with the authority and responsibility to act on the findings. 
- Process and structure. CI requires appropriate policies, procedures and an infrastructure so that employees may contribute effectively to the CI system as well as gain benefits from the CI process.

- Organizational awareness and culture. For a company to utilize its CI efforts successfully, an appropriate organizational awareness of CI and a culture of competitiveness must exist. While decision makers should determine what intelligence is required, information gathering should be on everyone's mind (Kahaner 1997).

\section{Dissemination}

The results of the CI process or project need to be disseminated to those with the authority and responsibility to act on the findings (Calof and Skinner 1999). Communication does, however rely on tools for communication, for example information collaboration tools, an Intranet and information sharing software and is dependent on a structure for CI and therefore these aspects are also discussed in this article.

Recent studies conducted into the CI practices of exporters, of which the results have not yet been published, have shown that South African companies do not fare well in terms of dissemination and process and structure - that little time is spent on dissemination or communication. Dissemination in CI consists of many aspects. It is about communicating intelligence to those who need to use it, it is about disseminating and sharing information in the company and it is about communicating the key focus areas (the key intelligence needs) to those people in the company who can constitute and should participate in the CI activity. Dissemination is also about having the right communication means, for example intelligence products, such as profiles on competitors, industries and customers, intelligence newsbriefs and making it easy for people to communicate information. Many companies, for example, have a central point to which employees can e-mail snippets of information they come across and that they think might be valuable.

The structure for CI justifies a column in itself but suffice to say that different companies have different CI structures and that there is no blue print for CI in this regard. CI being a strategic management tool, does, however, require some form of centralization (especially in multinational or large companies). In smaller companies, it is easier to manage CI from a central point although such companies typically would not have the luxury of resources, for CI requires a dedicated unit or dedicated people. Larger companies have been found to have success in conducting CI in a hub and spoke model with a small, effective centralized unit supported by decentralized spokes in various business units of or divisions in the company. Having a company-wide CI capability and company-wide participation is one of the critical success factors for CI.

Companies that have formalized the process of gathering, analysing and interpreting information have shown the best returns on investment in CI. Strategically managed companies demand continuous CI as input to management at all levels.

Dissemination is also about regular interaction by the CI unit/manager with the clients of intelligence, typically senior management, to determine their key intelligence needs and about communicating these focus points of CI to people in the company that can contribute in one way or another. These key intelligence needs are the drivers of CI and should be regularly tested to determine if they are still valid and should be derived from the strategic objectives of the company. They provide the focus for CI. Today's fast changing business environment also means that these key intelligence needs regularly change and, therefore, regular interaction (typically interviews) should take place with senior managers to validate or adapt the set of needs. 


\section{Format of intelligence products}

Regarding the products of CI, studies have shown that companies differ in the way they present intelligence. Culture, information, the client of the intelligence and the industry all are influencing factors on the way intelligence is package and presented. Typical products are competitor and industry profiles, intelligence alerts (used typically to convey urgent messages) and newsbriefs used to provide an overview of notable events and developments in a company's competitive environment. Intelligence estimates providing in-depth analyses, for example an industry, have high strategic value especially when they include scenarios and recommendations. Intelligence can also be communicated by means of e-mail, the Intranet, written documents, presentations or during meetings.

\section{Purpose of intelligence products}

A benchmarking study into CI conducted by the Special Libraries Association, suggests that CI products' main function should be to assist managers in solving a problem, in other words the intelligence should be actionable and it should be easy for the manager to understand the message and how to act upon it. It should therefore be timely, provide actionable findings and recommendations and must have integrity and credibility. CI's success is rooted in effectively disseminating intelligence and the format used to communicate intelligence should be appropriate for each end-user. Some prefer thick documents containing substantiating facts and reasoning while others prefer briefings, emails or presentations. CI expert Calof (1997) summarized the elements of useful intelligence results in the following manner:

- Resource-efficient. It is difficult to calculate the return on investment in CI partly because the benefit of CI is not immediately discernable. Resource input should cost less than the resultant output is worth. Often the costliest aspect is obtaining information and that is why it is useful to first find out, through conducting an information audit, what information is already available within the company. Effective communication of information could drastically lower the cost of information. Furthermore, when data being used in analysis come from primary sources (i.e. most human intelligence), it lowers the probable level of analytical accuracy, and it requires greater skill to elicit what is actually required from the primary sources. Nevertheless, many secondary databases may give great accuracy and timeliness but little in the way of a future-orientation while costing much and being also accessible to competitors.

- Objective. This relates to the presence of biases held by the analyst and/or company. To minimize the potentially destructive nature of these common biases, the data or information should be viewed and analysed using a rational and systematic approach. In other words, successful analysis minimizes the destructive potential of analytical and decision-oriented biases.

- Useful. Valuable intelligence output must be appropriate to the decision maker's responsibilities and company context as well as the intelligence need that was raised. The key for the analyst is to develop outputs on a 'need to know' and not 'nice to know' basis and that meet or surpass the client's critical intelligence needs. This criterion also suggests that the analytical outputs and process must be clearly communicated in a language that can be easily explained and understood by the analytical output recipient.

- Timely. Intelligence has a sell-by date but it is also important to remember that intelligence disseminated too early will also lose impact. Certain methods of analysis may provide the intelligence required but take far too long to develop. On the other hand, other methods of analysis may require little time but do not deliver the required features of objectivity, accuracy, utility, and resource efficiencies. Valuable analysis 
will provide decision makers enough time to allow the company to implement the course of action recommended by the analysis.

- Effective channels. CI professionals need efficient, flexible and thorough dissemination channels during the course of their work.

- Intelligence reports should also be predictive (what is going to happen, not what did happen) they should contain new insights, and provide reasons why events occur, not what happened. Intelligence reports should also spell out probably outcomes and recommendations. They should present the complete picture - not bits and pieces of the whole.

The Society of Competitive Intelligence Professionals lists a few competencies required for $\mathrm{CI}$ and it was thought appropriate to provide the complete list in this, the final column. The various competencies cover the CI areas of key intelligence needs, collection, analysis, CI management and, importantly, communication (SCIP Web site).

Competencies required for competitive intelligence

Prepared by the Society Competitive Intelligence Professionals

\section{Obtaining CI requests}

- Understand how to identify and elicit the intelligence needs of decision makers exactly.

- Develop effective communication, interviewing and presentation skills.

- Understand basic psychology types to appreciate the different orientations of decision makers.

- Know the company structure, culture and environment as well as the key 'informants'.

- Remain objective.

- Articulate key intelligence needs into the intelligence cycle.

- Know the internal and external capabilities.

- Conduct an information resource gap-analysis.

\section{Collecting information}

- Obtain knowledge of primary and secondary sources.

- Know the various methods for accessing internal and external, primary and secondary sources.

- Manage primary and secondary sources appropriately.

- Know how to execute the triangulation, multi-method, multi-source approach.

- Develop confidence level by ensuring reliability and validity of sources.

- Recognize anomalies in the information.

- Know the difference between hypothesized and open assumptions and why.

- Develop formal research skills.

- Recognize corporate information-gathering patterns and collect accordingly.

- Know the ethics associated with data gathering.

\section{Analysis and synthesis of information}

- Recognize the interaction between the collection and analysis phases.

- Analyse creatively.

- Employ inductive and deductive reasoning.

- Use network analysis, alternative thinking.

- Obtain an overview of basic analytical models.

- Introduce exciting and attractive models to elicit the discovery notion of analysis 
rather than the dry, research approach.

- Know when and why to use personality profiling, financial analysis, economic analysis, accounting analysis, trend analysis, risk assessment, quantitative and qualitative analysis, influence diagrams, opportunity analysis, pattern analysis, core vulnerabilities analysis, event analysis, linchpin analysis, etc.

- Recognize the inevitable existence of gaps and blind spots.

- Know when to cease analysing (analysis paralysis).

\section{Communicating Intelligence}

- Use persuasive presentation skills.

- Demonstrate empathy and use counselling skills, when appropriate.

- Organize findings and convey them with assertiveness and diplomacy.

- Use the format or media appropriate for each end-user.

- Recognize the effective volume and level of disseminating intelligence.

- Realise that listening can also be a form of presenting.

\section{Contextual and Management}

- Define the intelligence function.

- Explain how the intelligence cycle transforms information into intelligence.

- Explain the role of competitive intelligence within decision making, strategic planning and business development.

- Differentiate between competitive, competitor, business, technical and counter intelligence.

- Discuss the importance of a learning or knowledge-based company.

- Develop insights on how to identify the strengths, weaknesses, and biases regarding information sharing within a company.

- Present models for the structure and organization of a competitive intelligence unit and the pitfalls of various alternatives.

- Offer alternative structures depending upon company size.

- Discuss how to conduct a decision audit, an information audit and a knowledge audit and the importance of these insights.

- Present ways to keep current with advancements in information technology.

- Present methods for creating a competitive intelligence culture.

- Discuss ways to market or sell competitive intelligence within the company.

\section{References}

Calof, J., and Skinner, B. 1999. Government's Role in Competitive Intelligence: What's Happening in Canada? Competitive Intelligence Magazine 2(2):20-23.

Calof, J.L. 1997. Competitive intelligence handbook: it will change the way executives conduct their business prepared for the Canadian Food Bureau. Ottawa: IBIS Research.

Kahaner, L. 1997. Competitive intelligence: How to gather, analyse and use information to move your business to the top. New York: Simon \& Schuster

McGonagle, JJ., and Vella, CM. 1993. Outsmarting the Competition: Practical Approaches to Finding and Outsmarting the Competition. Naperville, IL: Sourcebooks.

\section{About the author}

Marié-Luce Muller is a consulting competitive intelligence analyst with IBIS Business and 
Information Services (Pty) Ltd, a leading Pretoria-based CI consultancy. She has a distinguished career in competitive intelligence. Her primary experience lies in assisting companies in honing their CI capabilities. She also performs tracking and scanning activities on behalf of companies. Marié-Luce has published many articles on competitive intelligence (CEO Magazine, Finance Week, Business Week, Beeld, Die Burger, and the South African Journal of Business Management), including an article on South Africa as an emerging CI player, which was published in an international publication of the Society of Competitive Intelligence Professionals (SCIP). She has also published a series of booklets on competitive intelligence (Nuts and Bolts business series, published by Knowledge Resources) and is a member of a research team participating in an international study of competitive intelligence practices among exporting companies. Previously, she was involved in research into the status of competitive intelligence practices in South Africa. A member of SCIP, she holds a postgraduate degree from the University of Stellenbosch.

\section{Disclaimer}

Articles published in SAJIM are the opinions of the authors and do not necessarily reflect the opinion of the Editor, Board, Publisher, Webmaster or the Rand Afrikaans University. The user hereby waives any claim he/she/they may have or acquire against the publisher, its suppliers, licensees and sub licensees and indemnifies all said persons from any claims, lawsuits, proceedings, costs, special, incidental, consequential or indirect damages, including damages for loss of profits, loss of business or downtime arising out of or relating to the user's use of the Website. 
ISSN 1560-683X

Published by InterWord Communications for Department of Information Studies, Rand Afrikaans University 